\title{
Correction to: Prevalence and risk factors associated with vertebral osteoporotic fractures in patients with rheumatoid arthritis
}

\author{
Jing-jing Tong ${ }^{1} \cdot$ Sheng-qian X ${ }^{1} \cdot$ He-xiang Zong ${ }^{1} \cdot$ Mei-juan Pan ${ }^{1} \cdot$ Yu-zhu Teng ${ }^{1} \cdot$ Jian-hua Xu ${ }^{1}$
}

Published online: 21 April 2020

(C) International League of Associations for Rheumatology (ILAR) 2020

Correction to: Clinical Rheumatology (2020) 39:357-364 https://doi.org/10.1007/s10067-019-04787-9

The authors regrets that the original published version of this article contained errors.

The mistake in Abstract and Table 1, the prevalence of VOPF in RA patients is mistakenly written as $20.2 \%$. The correct results should read as follows: Of 865 RA patients, the prevalence of OP and VOPF was $33.6 \%$ and $19.2 \%$, respectively.

The correct numbers of RA patients with or without VOPF in the Table 3 are as follows: The number of RA patients with VOPF is 166 , and the number of RA patients without VOPF is 699.

The online version of the original article can be found at https://doi.org/ 10.1007/s10067-019-04787-9

Sheng-qian Xu

xsqian-1112@163.com

1 Department of Rheumatology \& Immunology, The First Affiliated Hospital of Anhui Medical University, Hefei 230022, Anhui, China 